\title{
Controlling chemical reactions of a single particle
}

\author{
Lothar Ratschbacher, Christoph Zipkes, Carlo Sias` and Michael Köhl
}

Traditionally, chemical reactions have been investigated by tuning thermodynamic parameters, such as temperature or pressure. More recently, laser ${ }^{1}$ or magnetic field ${ }^{2}$ control methods have emerged to provide new experimental possibilities, in particular in the realm of cold collisions. The control of reaction pathways is also a critical component to implement molecular quantum information processing ${ }^{3}$. For these studies, single particles provide a clean and well-controlled experimental system. Here, we report on the experimental tuning of the exchange reaction rates of a single trapped ion with ultracold neutral atoms by exerting control over both their quantum states. We observe the influence of the hyperfine interaction on chemical reaction rates and branching ratios, and monitor the kinematics of the reaction products. These investigations advance chemistry with single trapped particles towards achieving quantum-limited control of chemical reactions and indicate limits for buffer-gas cooling of single-ion clocks.

The full control over all quantum mechanical degrees of freedom of a chemical reaction allows the identification of fundamental interaction processes and the steering of chemical reactions. This task is often complicated in heteronuclear systems by a multitude of possible reaction channels, which make theoretical treatments very challenging. Therefore, focussing on the best-controlled experimental conditions, such as using state-selected single particles and low temperatures, is crucial for the investigation of chemical processes at the most elementary level. The hybrid system of trapped atoms and ions offers key advantages in this undertaking. On the one hand, ion traps offer a large potential well depth to trap the reaction products for precision manipulation and investigation. On the other hand, contrary to pure ionic systems, there is no Coulomb barrier between the particles to fundamentally prevent chemical reactions at low temperatures. Therefore, the efforts to control the motional degrees of freedom of one $e^{4-6}$ and both ${ }^{7-13}$ reactants in hybrid atom-ion systems have paved new ways towards cold chemistry. The yet missing component is the simultaneous control of the internal degrees of freedom.

The interaction between an ion and a neutral atom at long distances is dominated by the attractive polarization interaction potential $V(r)$, which is of the form

$$
V(r)=-\frac{C_{4}}{2 r^{4}}
$$

Here, $C_{4}=\alpha_{0} q^{2} /\left(4 \pi \epsilon_{0}\right)^{2}$ is proportional to the neutral particle polarizability $\alpha_{0}, q$ is the charge of the ion, $\epsilon_{0}$ is the vacuum permittivity, and $r$ is the internuclear separation. Inelastic collisions take place at short internuclear distances. In the cold, semiclassical regime this requires collision energies above the centrifugal barrier ${ }^{14-16}$. Such processes are referred to as Langevin-type collisions and happen, even for cold collisions ${ }^{7,9}$, at an energyindependent rate $\gamma_{\text {Langevin }}=2 \pi \sqrt{C_{4} / \mu} n_{\mathrm{a}}$. Here $\mu$ is the reduced mass of the collision partners and $n_{\mathrm{a}}$ is the neutral atom density.

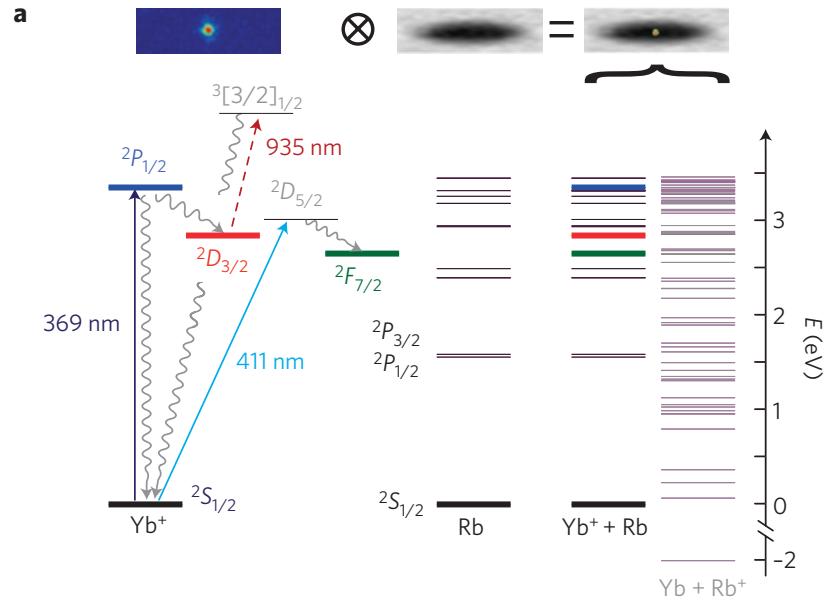

b

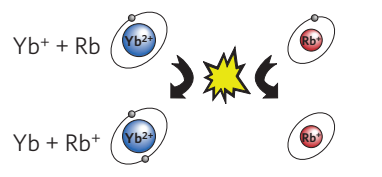

c
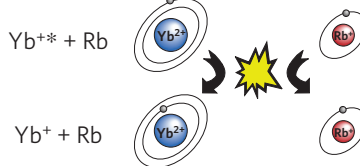

Figure 1 | Level scheme and inelastic processes of the ion-neutral system. a, Left: $\mathrm{Yb}^{+}$level scheme with the transitions used for optically pumping the ion (to scale). Middle: level scheme of a rubidium atom. Right: asymptotic level schemes of the two channels $\mathrm{Yb}^{+}+\mathrm{Rb}$ and $\mathrm{Yb}+\mathrm{Rb}^{+}$. The collision is initiated in the $\mathrm{Yb}^{+}+\mathrm{Rb}$ manifold and the $\mathrm{Yb}+\mathrm{Rb}^{+}$manifold can be populated by charge-exchange processes. $\mathbf{b}$, Pictorial representation of the charge exchange reaction, and, $\mathbf{c}$, quenching from an excited state. Shown are the filled core electronic shells and the relevant valence electrons. $\mathrm{Yb}^{+*}$ refers to an electronically excited state of $\mathrm{Yb}^{+}$.

More subtle effects, such as the hyperfine interaction, which may lead to atom-ion Feshbach resonances, are not included in the polarization potential and have been investigated only theoretically so far $^{17}$.

Experimentally, reactive Langevin collisions in the polarization potential have been investigated in ground state collisions ${ }^{7-10,12,18}$, which have exhibited relatively low rates for inelastic collisions, except for resonant charge exchange ${ }^{7}$. Recently, the first steps towards understanding reactive collisions in excited electronic states have been made using large ion crystals ${ }^{11-13}$, suggesting either a dominant contribution from very shortlived electronic states in the $\mathrm{Rb}+\mathrm{Ca}^{+}$system ${ }^{11}$ or, contrarily, a negligible contribution from excited state collisions in the $\mathrm{Ca}+\mathrm{Yb}^{+}$system $^{12}$.

Here, we demonstrate how control over the internal electronic state of a single ion and the hyperfine state of neutral atoms can be employed to tune cold exchange reaction processes. We study quenching, charge-exchange and branching ratios, and we use 
Table 1 | Measured proportionality constant $\epsilon$ and branching ratios.

\begin{tabular}{|c|c|c|c|c|c|c|}
\hline & ${ }^{2} S_{1 / 2}$ & ${ }^{2} D_{3 / 2}$ & ${ }^{2} F_{7 / 2}$ & ${ }^{2} P_{1 / 2}$ & ${ }^{2} S_{1 / 2}$ & ${ }^{2} D_{3 / 2}$ \\
\hline$\epsilon$ & $10^{-5 \pm 0.3}$ & $1.0 \pm 0.2$ & $0.018 \pm 0.004$ & $0.1 \pm 0.2$ & $(35 \pm 11) \epsilon_{\mathrm{S}}^{|2,2\rangle}$ & $1.0 \pm 0.2$ \\
\hline Charged particle lost & $65 \%$ & $87 \%$ & $84 \%$ & & $50 \%$ & \\
\hline $\mathrm{Rb}^{+}$identified & $35 \%$ & $12 \%$ & $15 \%$ & & $48 \%$ & \\
\hline Dark $\mathrm{Yb}^{+}$identified & & $<1 \%$ & & & $<1 \%$ & \\
\hline Hot ion (unidentified) & & & $1 \%$ & & $2 \%$ & \\
\hline Number of events & 283 & 754 & 225 & & 236 & \\
\hline
\end{tabular}

The columns $2-5$ of the table refers to neutral atoms in the $\left|F=2, m_{F}=2\right\rangle$ state and columns 6 and 7 to neutral atoms in the $\left|F=1, m_{F}=1\right\rangle$ state. Loss events of the charged particle are always chemical reactions for the $S$-states, whereas for $D$ - and $F$-states quenching and chemical reactions can contribute.

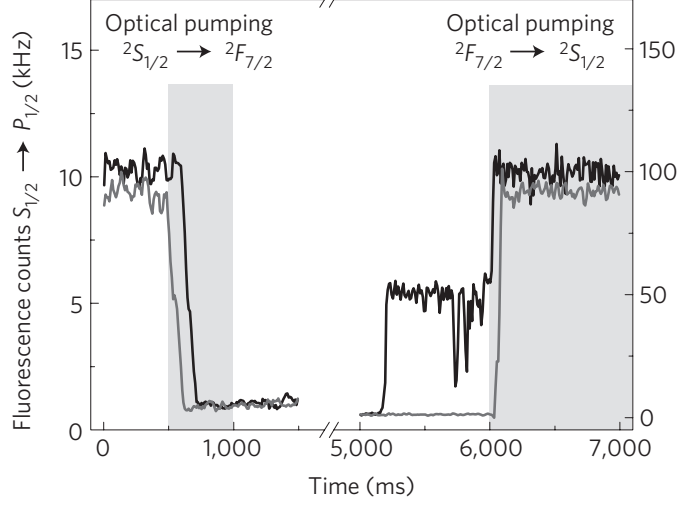

Figure 2 | Collisional quenching from ${ }^{2} F_{7 / 2}$ to ${ }^{2} S_{1 / 2}$. A two-ion Coulomb crystal is prepared in the ${ }^{2} F_{7 / 2}$ dark state by optical pumping, then interacts with the neutral atoms, and is subsequently probed by laser fluorescence (see Methods). An early appearance of laser fluorescence indicates collisional quenching (black curve) as compared to no quenching (grey curve). The large fluorescence dips indicate a high temperature of the ion crystal.

near-resonant laser light to control the rates. Our measurements show a high sensitivity of the charge-exchange reaction rates to the atomic hyperfine state, highlighting the influence of the nuclear spin on atom-ion collisions.

In our experiment we study collisions between ultracold ${ }^{87} \mathrm{Rb}$ atoms and single ${ }^{174} \mathrm{Yb}^{+}$ions, for which $\gamma_{\text {Langevin }} / n_{\mathrm{a}}=$ $2.1 \times 10^{-15} \mathrm{~m}^{3} \mathrm{~s}^{-1}$. We start by determining the inelastic collision loss rate coefficients for the long-lived ${ }^{2} D_{3 / 2}$ (radiative lifetime $52 \mathrm{~ms}$ ) and ${ }^{2} F_{7 / 2}$ (radiative lifetime 10 years) states of the ion (see Fig. 1 and Methods). The collisional stability of these states is of importance in buffer-gas-cooled ion clocks ${ }^{19}$ and quantum information processing. By optical pumping, both states are prepared as 'dark' states (see Methods), to study pure twobody collisions in the absence of light. This approach differs fundamentally from previous experiments in atom-ion ${ }^{11}$ excited state collisions, which always have been in presence of nearresonant laser light. We measure the inelastic loss rate $\gamma_{\ell}$ by immersing the ion for a variable time $t$ into the neutral atom cloud and determining the survival probability ${ }^{8,9,20,21} P_{\mathrm{s}}=\exp \left(-\gamma_{\ell} t\right)$. To understand and model our data, we make three assumptions: first, the atom and the ion can undergo an inelastic process only in a Langevin-type collision. Second, the characteristic collision time is significantly shorter than the radiative lifetime. Third, inelastic collisions can only be exothermic thanks to the very low kinetic energy of the colliding partners and the absence of resonant laser light. We model $\gamma_{\ell}=\epsilon \gamma_{\text {Langevin }}$ as proportional to the Langevin collision rate using the statedependent proportionality constant $\epsilon$. For the electronic ground state of the ion interacting with the $\left|F=2, m_{F}=2\right\rangle$ hyperfine ground state of $\mathrm{Rb}$ we find $\epsilon_{S}^{|2,2\rangle}=10^{-5 \pm 0.3}$, and for the excited states we measure $\epsilon_{D}^{|2,2\rangle}=1.0 \pm 0.2$ and $\epsilon_{F}^{|2,2\rangle}=0.018 \pm 0.004$ (see Table 1). The constant $\epsilon=1$ corresponds to the largest allowed inelastic collision rate in the semiclassical model, and even in near-resonant charge-exchange between equal elements of atoms and ions ${ }^{7}$ it was expected and approximately found to be $\epsilon=1 / 2$. In this regard, our results are unexpected, as the ${ }^{2} D_{3 / 2}$ state of $\mathrm{Yb}^{+}$combined with the ${ }^{2} S_{1 / 2}$-state of $\mathrm{Rb}$ is off-resonant by $0.14 \mathrm{eV}$ to the next available asymptotic state in the $\mathrm{Yb}+\mathrm{Rb}^{+}$manifold (see Fig. 1 ). In contrast, the ${ }^{2} F_{7 / 2^{-}}$ state has nearby asymptotic states but its inelastic collision rate is significantly lower.

We now compare these results with inelastic collisions in the absolute lowest hyperfine state $\left|F=1, m_{F}=1\right\rangle$ of the neutral atom. The hyperfine energy difference between the $\left|F=1, m_{F}=1\right\rangle$ and $\left|F=2, m_{F}=2\right\rangle$ states of $\mathrm{Rb}$ is $30 \mu \mathrm{eV}$, which is larger than the collision energy but negligible on the scale of the molecular potentials or the trap depth $(250 \mathrm{meV})$. We find a significantly enhanced probability for inelastic collisions with the ion in the electronic ground state $\epsilon_{S}^{|1,1\rangle}=(35 \pm 11) \times \epsilon_{S}^{|2,2\rangle}$, which demonstrates the important role of the hyperfine interaction. For collisions with the ion in the $D_{3 / 2}$ state, the value of $\epsilon$ remains unchanged.

For collisions in the electronic ground state of the ion, exothermic inelastic collisions are inevitably associated with a chemical reaction, leading to charge exchange or possibly the radiative association of molecules. The excess energy is converted into photons and/or kinetic energy of the reaction products. Depending on the amount of kinetic energy released, the reaction products are ejected from the ion trap or kept and detected by in-trap mass spectrometry ${ }^{9,22}$. A partial conversion of internal energy into kinetic energy in the predominantly predicted ${ }^{16}$ case of radiative charge exchange could be caused by the energy-dependent Franck-Condon overlap of the wave function of the entrance channels $\left(\mathrm{A}^{1} \Sigma^{+}\right.$ and $\left.\mathrm{a}^{3} \Sigma^{+}\right)$and the exit channel $\mathrm{X}^{1} \Sigma^{+}$of the $(\mathrm{RbYb})^{+}$potentials, owing to a $70 \%$ smaller polarizability of the $\mathrm{X}^{1} \Sigma^{+}$state (assuming negligible momentum transfer by the photon and constant orbital angular momentum). We control the distribution of the kinetic energy release in the charge exchange reaction by employing the hyperfine state of the neutral atoms; for atoms in $\left|F=1, m_{F}=1\right\rangle$ we retain $\mathrm{Rb}^{+}$with $(48 \pm 3) \%$ probability in the ion trap and for atoms in $\left|F=2, m_{F}=2\right\rangle$ we find it with $(35 \pm 3) \%$ probability. We also study inelastic collisions in the excited dark $D$ - and $F$-states, where the probabilities for observing $\mathrm{Rb}^{+}$are quite similar to each other (see Table 1). We do not observe the formation of $(\mathrm{YbRb})^{+}$ or $\mathrm{Rb}_{2}^{+}$molecules. The search for the latter was performed with the isotope ${ }^{176} \mathrm{Yb}^{+}$to achieve the required mass resolution.

With regards to collisional quenching between different electronic levels of the ion, we are principally able to detect two different quenching scenarios: ${ }^{2} F_{7 / 2} \rightarrow{ }^{2} S_{1 / 2}$ and ${ }^{2} D_{3 / 2} \rightarrow{ }^{2} F_{7 / 2}$. We directly observe quenching of ${ }^{2} F_{7 / 2} \rightarrow{ }^{2} S_{1 / 2}$ as shown in Fig. 2, where we exemplarily show two different experimental runs, with and without quenching (see also Methods). After the quenching, 


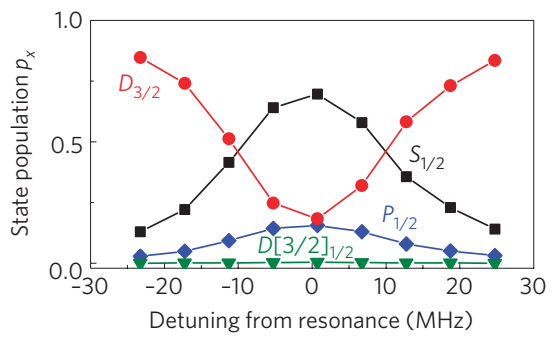

b $\approx$

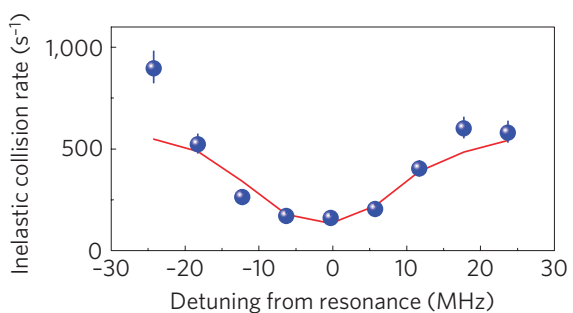

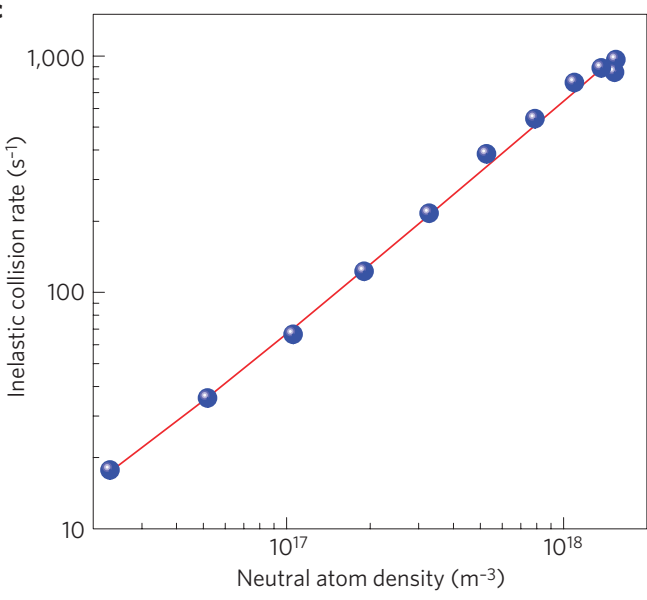

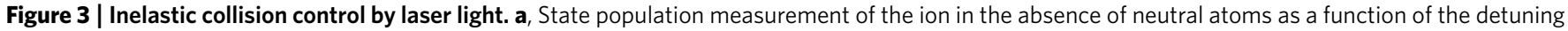
of the repump laser at $935 \mathrm{~nm}$. $\mathbf{b}$, Inelastic collision rate for the same detuning data in the presence of neutral atoms at $n_{\mathrm{a}}=1 \times 10^{18} \mathrm{~m}^{-3}$. The solid line shows the theoretical result of equation (1). c, Density dependence of the inelastic collision rate with the repump laser on resonance. The exponent of the power-law fit (solid line) is $0.98 \pm 0.02$.

we observe an increased temperature of the ions, which indicates a release of kinetic energy smaller than the depth of our trap, despite the large energy gap between the ${ }^{2} S_{1 / 2}$ and ${ }^{2} F_{7 / 2}$ states. The quenching rate from ${ }^{2} D_{3 / 2} \rightarrow{ }^{2} F_{7 / 2}$ was observed not to be detectable above our background rate.

Having established the inelastic collision parameters of the metastable $D$ - and $F$-states without resonant laser light, we now turn our attention to inelastic collisions in the presence of laser light for both the ${ }^{2} S_{1 / 2}-{ }^{2} P_{1 / 2}(369 \mathrm{~nm})$ and the ${ }^{2} D_{3 / 2}-{ }^{3} D[3 / 2]_{1 / 2}$ $(935 \mathrm{~nm})$ transitions. The purpose of the light is to experimentally tune the rates and to observe the occurrence of inelastic collisions in real time. The radiative lifetime of the ${ }^{2} P_{1 / 2}$ state $(8 \mathrm{~ns})$ is too short compared with the collision rate to provide pure $P$-state measurements. Here and in the following, we assume that the $F$-state is unoccupied, because the 411-nm light is turned off and collisional quenching into the $F$-state has been measured to be negligible. Therefore, the overall inelastic collision rate is determined by a mixture of $S_{-}, P_{-}, D_{-}$, and $D[3 / 2]$-states

$$
\gamma_{\ell}=2 \pi \sqrt{C_{4} / \mu} n_{\mathrm{a}}\left(p_{S} \epsilon_{S}+p_{P} \epsilon_{P}+p_{D} \epsilon_{D}+p_{D[3 / 2]} \epsilon_{D[3 / 2]}\right)
$$

Here, $p_{x}$ is the occupation probability of state $x$, which we determine experimentally for different settings of laser intensities and detunings (see Methods). In Fig. $3 a$ we show the state populations of the $S, P, D$ and ${ }^{3} D[3 / 2]_{1 / 2}$ states as we vary the frequency of the laser at $935 \mathrm{~nm}$ on the ${ }^{2} D_{3 / 2}{ }^{3} D[3 / 2]_{1 / 2}$ transition. Figure $3 \mathrm{~b}$ shows the associated change in the inelastic collision rate in the presence of the neutral atoms. We demonstrate tuning by one order of magnitude and we find it closely follows the model of equation (1) indicating a dominant contribution from the $D$-state. From these data we also extract $\epsilon_{P}=0.1 \pm 0.2$, which is small and consistent with zero. Figure $3 c$ shows the linear scaling of the inelastic collision rate with neutral atom density, confirming the picture of binary collisions.

Finally, we turn our attention to the observation of the kinematics of the collision products. In Fig. 4 we show the fluorescence at $369 \mathrm{~nm}$ during the interaction, with the $935-\mathrm{nm}$ light on. Figure $4 a-c$ shows individual experimental runs, in which the initial sharp loss of fluorescence results from an inelastic collision. If some kinetic energy is released in this process, the

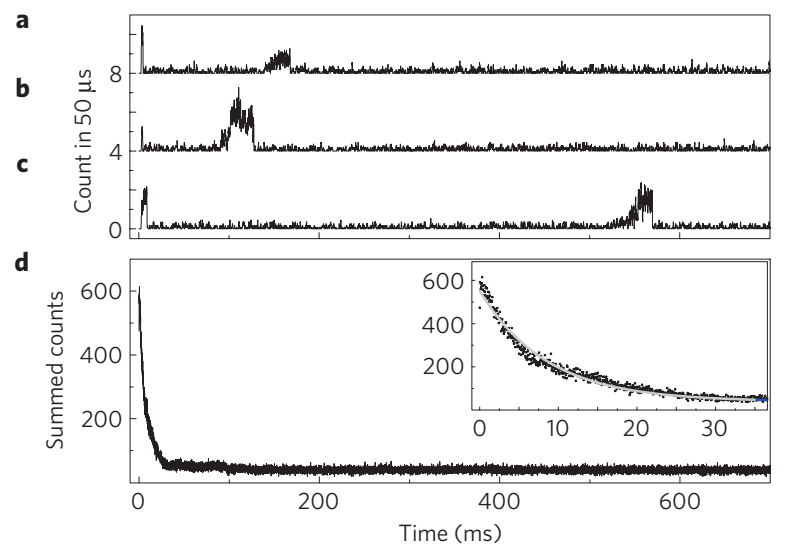

Figure 4 | Monitoring of inelastic atom-ion collisions. a-c, Recorded fluorescence for selected events. After a quick initial loss, the fluorescence reoccurs after random times when the kinetic energy released in the inelastic collision has been removed by cooling. Then, a second collision occurs and the ion disappears again. The curves are vertically offset for clarity. d, Sum of 343 repetitions of the experiment, which is fitted with an exponential decay for short times. Inset, zoom-in on the initial decay of plot $\mathbf{d}$. The solid line is an exponential fit to the data.

ion is on a large trajectory in the trap (much larger than the size of the atom cloud), but can be re-cooled, which is signalled by the relatively slow increase of the reoccurring fluorescence, from which we determine a lower bound for the release energy of $8 \mathrm{meV}$. We observe in $4 \%$ of our events that the fluorescence of the ion reoccurs after a certain time, before it undergoes a second inelastic collision and is dark again. As we keep the $\mathrm{Yb}^{+}$ ion in the trap, these processes are not charge-exchange reactions but quenching processes with a kinetic energy release less than $\approx 250 \mathrm{meV}$. Because the ion is colliding in highly excited electronic states, such as $P_{1 / 2}$ and $D_{3 / 2}$ with internal energies of $\approx 3 \mathrm{eV}$, this suggests a mostly radiative decay into the ground state $S_{1 / 2}$. We have ruled out that the reoccurrence events are linked to the dissociation of a potential molecular ion in a secondary collision with neutral atoms ${ }^{12}$. To this end, we have performed this measurement also with extremely short interaction times between neutral atoms and 
the ion (on the order of a few collision times) and performed mass spectrometry on the dark ion after the collision, confirming the absence of a molecular ion.

\section{Methods}

Preparation of ultracold atoms and ions. We prepare $4 \times 10^{5}$ neutral ${ }^{87} \mathrm{Rb}$ atoms in the $\left|F=2, m_{F}=2\right\rangle$ hyperfine state of the electronic ground state at temperature down to $T \approx 200 \mathrm{nK}$ in a harmonic magnetic trap of characteristic frequencies ${ }^{23}$ $\left(\omega_{x}, \omega_{y}, \omega_{z}\right)=2 \pi \times(8,26,27) \mathrm{Hz}$. By changing atom number and temperature, we tune the atomic density by two orders of magnitude. The atoms can be transferred into an optical dipole trap formed by two crossed laser beams at 1,064 $\mathrm{nm}$. Here, the atoms can be transferred into the ground hyperfine state $\left|F=1, m_{F}=1\right\rangle$ using a resonant microwave pulse. At the same location, we trap single $\mathrm{Yb}^{+}$ions in a radio-frequency Paul trap with secular trap frequencies of $\omega_{\perp}=2 \pi \times 150 \mathrm{kHz}$ radially and $\omega_{\mathrm{ax}}=2 \pi \times 42 \mathrm{kHz}$ axially ${ }^{8,9}$. We use standard techniques to cool and detect single ions or small ion crystals on the cycling transition ${ }^{2} S_{1 / 2}-{ }^{2} P_{1 / 2}$ near $369 \mathrm{~nm}$ wavelength (see Fig. 1). From the excited ${ }^{2} P_{1 / 2}$ state, radiative decay populates the ${ }^{2} D_{3 / 2}$ state with a probability ${ }^{24}$ of $\approx 1 / 200$. For efficient laser cooling and detection, a laser at $935 \mathrm{~nm}$ pumps the population via the ${ }^{3} D[3 / 2]_{1 / 2}$ state back into the cooling cycle. Preparation in ${ }^{2} D_{3 / 2}$ (radiative lifetime $52 \mathrm{~ms}$ ) is achieved by optical pumping from the $S_{1 / 2}$ state using laser light at $369 \mathrm{~nm}$ in less than $10 \mu$ s in the absence of light at $935 \mathrm{~nm}$, which is significantly faster than the inverse of the Langevin collision rate of a few $10^{3} \mathrm{~s}^{-1}$. Alternatively, the ${ }^{2} F_{7 / 2}$ state (radiative lifetime 10 years) is populated by optical pumping on the ${ }^{2} S_{1 / 2}-{ }^{2} D_{5 / 2}$ line with spontaneous decay on the ${ }^{2} D_{5 / 2}-{ }^{2} F_{7 / 2}$ transition ${ }^{25}$. The ion is spatially overlapped with the centre of the ultracold neutral atom cloud by displacing the two independent trapping potentials. The typical collision energies ${ }^{26}$, due to residual micromotion, are on the order of $\sim 0.004 \mathrm{meV}$.

Detection of collisional quenching. We prepare two ions in a small Coulomb crystal at time $t=0$ in the ${ }^{2} S_{1 / 2}$ ground state, which we detect by near-resonant laser fluorescence at $369 \mathrm{~nm}$ (together with repumping at $935 \mathrm{~nm}$ ). The optical pumping into the ${ }^{2} F_{7 / 2}$ state using light at $411 \mathrm{~nm}$ is switched on at $t=500 \mathrm{~ms}$ for $500 \mathrm{~ms}$ and, typically within $100 \mathrm{~ms}$, the ion is pumped into the desired state (see Fig. 2). As a result, the fluorescence counts on the $369 \mathrm{~nm}$ transition drop to zero. After the interaction with the neutral atoms (effective duration typically $16 \mathrm{~ms}$ ) and the subsequent removal of the neutral atom cloud, the fluorescence on the ${ }^{2} S_{1 / 2}-{ }^{2} P_{1 / 2}$ transition (together with repumping at $935 \mathrm{~nm}$ ) is probed from $t=5,000 \mathrm{~ms}$ onwards. If the ion is still in the ${ }^{2} F_{7 / 2}$ state it will not scatter photons however, if it has been quenched to ${ }^{2} S_{1 / 2}$, we observe fluorescence. At $t=6,000 \mathrm{~ms}$ the ions are optically pumped from the $F$-state back into the $S$-state to ensure that no ions have been lost. The fluorescence count rate during the preparation part of the sequence is lower owing to the presence of an offset field from the magnetic atom trap, which is turned off when the atoms are released.

Experimental determination of the ion electronic state occupation. Owing to the experimental situation of a multilevel system in the presence of a magnetic field and imperfect polarization of the laser beams, we determine the state populations $p_{x}=\tau_{x} / \tau_{c}$ experimentally rather than relying on theoretical modelling. Here, $\tau_{c}$ is the average time between two spontaneous decays on the ${ }^{2} P_{1 / 2}-{ }^{2} D_{3 / 2}$ transition, $\tau_{x}$ is the average time spent in state $x \in\{S, P, D, D[3 / 2]\}$ during $\tau_{\mathrm{c}}$, and $\tau_{\mathrm{c}}=\sum_{x} \tau_{x}$. The time $\tau_{P}$ is given by the decay rate $\Gamma$ of the $P_{1 / 2}$ state and the branching ratio of the $P$-state into $S$ - and $D$-states as $\tau_{P}=200 / \Gamma$. A sequence of alternating pulses from the $369 \mathrm{~nm}$ and $935 \mathrm{~nm}$ lasers are used to determine $\tau_{S}$ and $\tau_{D} . \tau_{P}+\tau_{S}$ is observed as the exponential decay constant of fluorescence after the $369 \mathrm{~nm}$ laser is pulsed on. The pulse length is set to get complete depletion of the $S-P$ system into the $D$-state. For a given setting of intensity and detuning of the 935-nm laser, the average number of photons $N_{369}$ per $369 \mathrm{~nm}$ pulse depends on the duration $t_{935}$ of the preceding repump pulse. Varying the length $t_{935}$ allows us to retrieve $\tau_{D}$ and the photon detection efficiency $\eta$ from the fit of $200 \eta\left[1-\exp \left(-t_{935} / \tau_{D}\right)\right]=N_{369}$ to the counted photon number. We find $\eta=(2.1 \pm 0.1) \times 10^{-3}$, in accordance with the numerical aperture of the imaging system and the quantum efficiency of the single-photon counter. The lifetime of the excited ${ }^{3} D[3 / 2]_{1 / 2}$ state is $\tau_{D[3 / 2]}=40 \mathrm{~ns}$ and the branching ratio is $98 \%$ into $S_{1 / 2}$ and $2 \%$ into $D_{3 / 2}$. Decay into $P_{1 / 2}$ is not dipole-allowed owing to parity ${ }^{27}$ and therefore we do not have to consider a cascade through intermediate levels.

\section{Received 5 April 2012; accepted 20 June 2012; published online} 22 July 2012

\section{References}

1. Jones, K. M., Tiesinga, E., Lett, P. D. \& Julienne, P. S. Ultracold photoassociation spectroscopy: Long-range molecules and atomic scattering. Rev. Mod. Phys. 78, 483-535 (2006).

2. Chin, C., Grimm, R., Julienne, P. \& Tiesinga, E. Feshbach resonances in ultracold gases. Rev. Mod. Phys. 82, 1225-1286 (2010).
3. DeMille, D. Quantum computation with trapped polar molecules. Phys. Rev. Lett. 88, 067901 (2002)

4. Willitsch, S., Bell, M. T., Gingell, A. D., Procter, S. R. \& Softley, T. P. Cold reactive collisions between laser-cooled ions and velocity-selected neutral molecules. Phys. Rev. Lett. 100, 043203 (2008).

5. Staanum, P. F., Højbjerre, K., Wester, R. \& Drewsen, M. Probing isotope effects in chemical reactions using single ions. Phys. Rev. Lett. 100, 243003 (2008).

6. Roth, B., Blythe, P., Wenz, H., Daerr, H. \& Schiller, S. Ion-neutral chemical reactions between ultracold localized ions and neutral molecules with single-particle resolution. Phys. Rev. A 73, 042712 (2006)

7. Grier, A. T., Cetina, M., Oručević, F. \& Vuletić, V. Observation of cold collisions between trapped ions and trapped atoms. Phys. Rev. Lett. 102, 223201 (2009).

8. Zipkes, C., Palzer, S., Sias, C. \& Köhl, M. A trapped single ion inside a Bose-Einstein condensate. Nature 464, 388-391 (2010).

9. Zipkes, C., Palzer, S., Ratschbacher, L., Sias, C. \& Köhl, M. Cold heteronuclear atom-ion collisions. Phys. Rev. Lett. 105, 133201 (2010).

10. Schmid, S., Härter, A. \& Hecker Denschlag, J. Dynamics of a cold trapped ion in a Bose-Einstein condensate. Phys. Rev. Lett. 105, 133202 (2010).

11. Hall, F. H. J., Aymar, M., Bouloufa-Maafa, N., Dulieu, O. \& Willitsch, S. Light-assisted ion-neutral reactive processes in the cold regime: Radiative molecule formation versus charge exchange. Phys. Rev. Lett. 107, 243202 (2011).

12. Rellergert, W. G. et al. Measurement of a large chemical reaction rate between ultracold closed-shell ${ }^{40} \mathrm{Ca}$ atoms and open-shell ${ }^{174} \mathrm{Yb}^{+}$ions held in a hybrid atom-ion trap. Phys. Rev. Lett. 107, 243201 (2011).

13. Sullivan, S. T., Rellergert, W. G., Kotochigova, S. \& Hudson, E. R. The role of electronic excitation in cold atom-ion chemistry. Preprint at http://arxiv.org/abs/1205.4286 (2012).

14. Langevin, P. A fundamental formula of kinetic theory. Ann. Chim. Phys. 5, 245-288 (1905).

15. Vogt, E. \& Wannier, G. H. Scattering of ions by polarization forces. Phys. Rev. 95, 1190-1198 (1954)

16. Makarov, O. P., Côté, R., Michels, H. \& Smith, W. W. Radiative charge-transfer lifetime of the excited state of $\mathrm{NaCa}^{+}$. Phys. Rev. A 67, 042705 (2003).

17. Idziaszek, Z., Calarco, T., Julienne, P. S. \& Simoni, A. Quantum theory of ultracold atom-ion collisions. Phys. Rev. A 79, 010702 (2009).

18. Ravi, K., Lee, S., Sharma, A., Werth, G. \& Rangwala, S. A. Sympathetic and swap cooling of trapped ions by cold atoms in a MOT. Preprint at http://arxiv.org/abs/1112.5825 (2011).

19. Schauer, M. M. et al. Collisional population transfer in trapped $\mathrm{Yb}^{+}$ions. Phys. Rev. A 79, 062705 (2009).

20. Ueberholz, B., Kuhr, S., Frese, D., Meschede, D. \& Gomer, V. Counting cold collisions. J. Phys. B 33, L135-L142 (2000).

21. Weber, C., John, S., Spethmann, N., Meschede, D. \& Widera, A. Single Cs atoms as collisional probes in a large $\mathrm{Rb}$ magneto-optical trap. Phys. Rev. A 82, 042722 (2010).

22. Drewsen, M., Mortensen, A., Martinussen, R., Staanum, P. \& Sørensen, J. L. Nondestructive identification of cold and extremely localized single molecular ions. Phys. Rev. Lett. 93, 243201 (2004).

23. Palzer, S., Zipkes, C., Sias, C. \& Köhl, M. Quantum transport through a Tonks-Girardeau gas. Phys. Rev. Lett. 103, 150601 (2009).

24. Olmschenk, S. et al. Manipulation and detection of a trapped $\mathrm{Yb}^{+}$hyperfine qubit. Phys. Rev. A 76, 052314 (2007).

25. Taylor, P. et al. Investigation of the ${ }^{2} S_{1 / 2}-{ }^{2} D_{5 / 2}$ clock transition in a single ytterbium ion. Phys. Rev. A 56, 2699-2704 (1997).

26. Zipkes, C., Ratschbacher, L., Sias, C. \& Köhl, M. Kinetics of a single trapped ion in an ultracold buffer gas. New J. Phys. 13, 053020 (2011).

27. Bièmont, E., Dutrieux, J., Martin, I. \& Quinet, P. Lifetime calculations in Yb II J. Phys. B 31, 3321-3333 (1998).

\section{Acknowledgements}

We thank D. Sigle for experimental assistance, Engineering and Physical Sciences Research Council (EP/H005676/1), European Research Council (Grant number 240335), and the Leverhulme Trust (C.S.) for support.

\section{Author contributions}

The experimental protocols were devised by all authors. Data taking and data analysis was performed by L.R., C.Z. and C.S. The manuscript was written by M.K. with contributions from all authors.

\section{Additional information}

Reprints and permissions information is available online at www.nature.com/reprints. Correspondence and requests for materials should be addressed to C.S.

\section{Competing financial interests}

The authors declare no competing financial interests. 